\title{
Impacto de la virtualidad en las prácticas preprofesionales de la carrera Desarrollo Infantil Integral: caso de un Instituto Superior Tecnológico
}

\section{Impact of virtuality in the pre-professional practices of the Comprehensive Child Development career: case of a Higher Technological Institute}

\author{
Nazly Micaela Veloz Rodríguez \\ Instituto Superior Tecnológico Tsáchila, Santo Domingo, Ecuador \\ nazlyrodriguezveloz@tsachila.edu.ec \\ https://orcid.org/0000-0002-6783-4224 \\ Cristopher David Herrera Navas \\ Pontificia Universidad Católica del Ecuador, Santo Domingo, Ecuador \\ cdherreran@pucesd.edu.ec \\ https://orcid.org/0000-0002-2031-5187
}

Recepción: 22/03/2021 | Aceptación: 25/05/2021 | Publicación: 10/09/2021

Cómo citar (APA, séptima edición):

Veloz Rodríguez, N. M., y Herrera Navas, C. D. (2021). Impacto de la virtualidad en las prácticas preprofesionales de la carrera Desarrollo Infantil Integral: caso de un Instituto Superior Tecnológico. INNOVA Research Journal, 6(3), 98-120. https://doi.org/10.33890/innova.v6.n3.2021.1727

\begin{abstract}
Resumen
La emergencia sanitaria provocada por el COVID-19 ha ocasionado que todos los ambientes de formación humana se dirijan hacia la virtualidad, entre ellos, se encuentran las prácticas preprofesionales, que resultan imprescindibles para los estudiantes de Institutos Superiores Tecnológicos. La presente investigación tuvo como propósito determinar el impacto de la virtualidad en la práctica preprofesional desde la perspectiva estudiantil y docente de la carrera Desarrollo Infantil Integral del Instituto Superior Tecnológico Tsáchilas en el año 2020. El enfoque investigativo es cualitativo, con diseño no experimental-transversal, de tipo descriptivo. La población está conformada por 45 estudiantes y dos docentes (coordinadora de carrera y de prácticas preprofesionales). Se utilizó como técnicas para la recolección de datos al grupo focal y a la entrevista, teniendo como instrumentos; cuestionario semiestructurado y entrevista semiestructurada. Los resultados demuestran que el impacto de la virtualidad desde la perspectiva
\end{abstract}


estudiantil fue negativo, ya que mencionan haber tenido dificultades recursivas, problemas de asignación e incapacidad para planificar e impartir clases desde la virtualidad. Por otro lado, los docentes mencionan tener una perspectiva positiva de la práctica preprofesional realizada, ya que la virtualidad permite mayor flexibilidad y beneficios, dando paso a nuevas posibilidades para la práctica pedagógica. Se concluye en que el impacto de la virtualidad en la calidad de la práctica preprofesional fue negativo y se necesita implementar medidas que partan del desarrollo de conciencia de los docentes acerca del problema a través de una comunicación asertiva y responsiva.

Palabras claves: educación; COVID-19; prácticas pre profesionales.

\begin{abstract}
The health emergency caused by COVID-19 has caused all human training environments to move towards virtuality, among them are pre-professional practices, which are essential for students of Higher Technological Institutes. The purpose of this research was to determine the impact of virtuality in pre-professional practice from the student and teacher perspective of the Comprehensive Child Development career of the Tsachila Higher Technological Institute in 2020. The research approach is qualitative, with a non-experimental design -transversal, descriptive type. The population is made up of 45 students and two teachers (coordinator of career and preprofessional practices). The focal group and the interview were used as techniques for data collection, having as instruments; semi-structured questionnaire and semi-structured interview. The results show that the impact of virtuality from the student perspective was negative, since they mention having had recursive difficulties, assignment problems and inability to plan and teach classes from virtuality. On the other hand, teachers mention having a positive perspective of the pre-professional practice carried out, since virtuality allows greater flexibility and benefits, giving way to new possibilities for pedagogical practice. It is concluded that the impact of virtuality on the quality of pre-professional practice was negative and it is necessary to implement measures that start from the development of teachers' awareness about the problem through assertive and responsive communication.
\end{abstract}

Keywords: education; COVID-19; pre professional practice.

\title{
Introducción
}

Existe evidencia variada de las repercusiones causadas por la emergencia sanitaria en todas las áreas con las que se vinculan el ser humano, entre ellas, la educación. En lo internacional, La Organización de las Naciones Unidas para la Educación, Ciencia y Cultura (UNESCO) (2020) menciona que más del 91,3\% (1,575,270,054) de estudiantes, han sido afectados por el cierre temporal de las instituciones educativas, las causas en la mayoría de los casos se originan por la carencia de recursos tecnológicos o falta de dominio de las herramientas que ofrecen y el precario acompañamiento que brindan los padres ante las actividades de estimulación.

De manera análoga, el problema que ha generado la emergencia sanitaria también se puede divisar en todo el continente americano, referente a ello el Fondo de las Naciones Unidas para la Infancia (UNICEF) (2020) expone que, pese a los mecanismos de contingencia implementados para el desarrollo de la educación, en la actualidad cerca de 1.200 millones de niños siguen afectados por el cierre de las escuelas, viéndose obligados a educarse en casa, esto genera desigualdades inherentes, debido que, no todos tienen acceso a las Tics y a las herramientas que 
proporcionan. Se advierte que esto agravaría la crisis existente en el continente referente a los procesos de formación humana.

Contextualizando el problema a nivel del Ecuador y haciendo referencia al desarrollo de las prácticas y a aplicación de competencias profesionales de educación en la virtualidad, está Guarnizo (2018) quien menciona que para la puesta en experiencia a través de las TICs de los conocimientos adquiridos en la formación universitaria es necesaria una especialización que adapte a los estudiantes al nuevo entorno laboral. Esto alude a la necesidad de un proceso de formación sistemático previo para la realización de las prácticas preprofesional en el campo virtual. Sin embargo, debido a llegada repentina de la emergencia sanitaria no se pudo realizar el debido proceso de especialización para la aplicación de las competencias laborales en entornos virtuales.

Asimismo, sucede en Santo Domingo de los Tsáchilas, específicamente en el Instituto Superior Tecnológico Tsáchilas en la carrera de Desarrollo Infantil Integral, donde la emergencia sanitaria ha obligado a las carreras de formación dual a realizar la puesta en práctica de las competencias a través de medios virtuales. Entonces, se han presentado diversas problemáticas que, de una u otra forma, repercuten la calidad del proceso de enseñanza-aprendizaje, entre ellas están: la falta de capacitación referente al traslado de los procesos educativos a las Tics, disponibilidad de tiempo de las familias para el desarrollo de las actividades de aprendizaje, la inexistencia de recursos didácticos y tecnológicos que contribuyan a los infantes, la ausencia de colaboración y compromiso por parte de las familias en los procesos estimulación infantil. Por ende, repercute en progreso de sus habilidades en las diferentes áreas de aprendizaje en los niños/as.

Sim embargo, la relevancia del aseguramiento en la calidad de la práctica preprofesional no es reciente, existen diversos estudios abordan la importancia de la práctica preprofesional en la formación del docente en educación inicial y la incorporación de nuevas herramientas que favorezcan la apropiación de competencias laborales, haciendo referencia a lo antes dicho se encuentra Cornejo (2014) con su estudio denominado "Prácticas profesionales durante la formación inicial docente: análisis y optimización de sus aportes a los que aprenden y a los que enseñan a aprender a enseñar" (p. 239). Teniendo como objetivo implementar políticas institucionales para la transformación de la práctica del docente de educación inicial. A través de una metodología de enfoque cualitativo, de tipo descriptiva, obtiene como principales resultados que: los enfoques más recientes de formación superior apuntan a relaciones mayormente asimétricas e interactivas, a partir de nuevos medios, entre la teoría y la práctica. Por lo que, se concluye que en el medio pedagógico existe carencia de programas y políticas innovadoras para la formación de formadores asociando la teoría y la práctica.

Por otro lado, aludiendo a la necesidad innovadora de buenos procedimientos educativos en entornos virtuales está García, Guerrero y Granados (2015) con su trabajo titulado "Buenas prácticas en los entornos virtuales de enseñanza-aprendizaje" (p.76). A través de una metodología de enfoque cualitativo y tipología documental, teniendo como finalidad la identificación de buenas prácticas docentes utilizadas en entornos de formación virtual, obtienen como principal resultado que existen numerosas herramientas virtuales desarrolladas por docentes a través de estrategias contextualizadas al medio y buenas prácticas para la consecución de un objetivo formativo. Como 
conclusión se obtiene que, a través de la aplicación de buenas prácticas profesionales, el promedio de los estudiantes es mayor en entornos totalmente virtuales, del mismo modo, la mayor parte de estudiantes se sienten cómodos trabajando en los EVEA (entornos virtuales de enseñanzaaprendizaje).

Del mismo modo, abordando los elementos que influyen en el desarrollo de la práctica pre profesional, se encuentra a Ruilova (2019) con su estudio titulado "Las prácticas preprofesionales en la formación inicial docente de EGB, el rol del maestro tutor" (p.15). Con el objetivo de determinar la influencia que tienen los maestros tutores de aula en la formación inicial de docentes de Educación General Básica, a través de una metodología de enfoque cualitativo de tipo documental, obteniendo como principales resultados que la muestra de motivación en el desarrollo de la labor educativa por parte de los docentes tutores contribuye a la apropiación de competencias profesionales en las prácticas preprofesionales, concluyendo así que, que la actualización y capacitaciones continuas docentes mejora la calidad educativa de las prácticas preprofesionales.

Continuando con la línea temática referente a la práctica preprofesional y sus implicaciones en la formación de docentes especializados en la primera infancia está Herrera (2019) con su investigación titulada "Evaluación de impacto de las prácticas pre profesionales en los graduados de la escuela de Ciencias de la Educación” (p.1). Mediante un constructo metodológico de enfoque cuantitativo, diseño no experimental de tipo transversal y alcance exploratorio, a partir del objetivo evaluar el impacto de las prácticas pre profesionales en los graduados de la escuela de Ciencias de la Educación, obtiene como resultados principales que el 50\% de estudiantes está medianamente satisfecho con el aporte de la práctica preprofesional a su desempeño docente, del mismo modo, el $50 \%$ de estudiantes con correcto desempeño docente obtiene un nivel de transferencia excelente en dicho proceso. De este modo, se concluye que la práctica preprofesional tiene un alto impacto en la formación de educadores.

A consecuencia de la importancia de la práctica preprofesional, se han desarrollado modelos para mejorar su desarrollo y, a consecuencia de ello, sistematizar los procesos y aprendizajes teóricos abordados en la práctica educativa, de ello hace referencia Rodríguez, Calle y Zabala (2018) en su estudio denominado "Modelo para desarrollar prácticas preprofesionales reflexivas en la formación de docentes para educación básica" (p.22). Con el objetivo de proponer un modelo de para el desarrollo y reflexión de la práctica preprofesional docente, a través de una metodología cualitativa, de revisión bibliográfica de tipo descriptiva-explicativa, se expone que la mayor parte de estudiantes poseen limitaciones para la involucración de las Tecnologías de la Información y de la Comunicación (Tics) en el desarrollo de la práctica preprofesional, asimismo, los docentes que supervisan la práctica han determinado deficiencias en el dominio de contenido y en la preparación pedagógica para brindar respuestas asertivas a problemas áulicos.

Los autores antes mencionados, a manera de conclusión, exponen que el modelo de práctica preprofesional que favorezca a la formación de los futuros docentes debe considerar los siguientes principios: a) De unidad dialéctica entre actividad y comunicación; b)De unidad en la relación de los procesos cognitivos y afectivos; c) De relación directa entre lo instructivo, educativo y formativo; d) De vinculación entre los saberes enseñados y las necesidades de la sociedad; e) De constante autorreflexión docente; f) De constante reflexión colectiva; g) De modelos pedagógicos activos, protagónicos e innovadores. 
En todas las investigaciones antes abordadas se estudia la importancia de la práctica preprofesional en la formación de docentes, algunos de los factores condicionantes de la calidad de la práctica, la necesidad de incorporar nuevas herramientas para el desarrollo de la práctica preprofesional y algunas estrategias y modelos cuya finalidad es asegurar las buenas prácticas docentes, sin embargo dentro de la investigación no se halló estudios que determinen la influencia de la virtualidad en el desarrollo de la práctica preprofesional docente. Por ello, el objetivo del presente estudio es: determinar el impacto de la virtualidad en la práctica preprofesional desde la perspectiva estudiantil y docente de la carrera Desarrollo Infantil Integral del Instituto Superior Tecnológico Tsáchilas en el año 2020-01.

Mediante el análisis elaborado sobre los antecedentes, problemática y marco teórico de la temática, se plantea un sistema de hipótesis con dos vías, las cuales son: hipótesis de investigación (Hi): la virtualidad tuvo un impacto negativo en la práctica pre profesional docente de los estudiantes de la carrera Desarrollo Infantil Integral del Instituto Superior Tecnológico Tsáchilas en el año 2020-01; hipótesis nula (H0) la virtualidad no tuvo un impacto negativo en la práctica pre profesional docente de los estudiantes de la carrera Desarrollo Infantil Integral del Instituto Superior Tecnológico Tsáchilas en el año 2020-01

\section{Marco Teórico}

\section{La virtualidad}

La virtualidad es aquella apariencia de la realidad, que se encuentra definida como un proceso imaginario, y que precisa un sistema de cómputo para objetivarse.

La palabra virtual procede del latín medieval virtualis, que a su vez deriva de virtus: fuerza, potencia. En la filosofía escolástica, lo virtual es aquello que existe en potencia, pero no en acto. Lo virtual tiende a actualizarse, aunque no se concretiza de un modo efectivo o formal. Con todo rigor filosófico, lo virtual no se opone a lo real sino a lo actual: virtualidad y actualidad sólo son dos maneras de ser diferentes. (Martínez, Leyva, Félix, Cecenas y Ontiveros, 2014, p.9)

El concepto, es muy usado en el campo de la informática y tecnología, con el objetivo de manifestar a la realidad edificada mediante sistemas o procesos digitales. Dentro de este marco, se conoce como realidad virtual al mecanismo informático que posibilita factiblemente a los navegadores para que puedan estar inmersos en un mundo paralelo al real. Cabe recalcar que el concepto de virtual es un proceso inverso a la actualización, por el hecho de ser una estructura que favorece a los procesos de creación (Delgado, 2019).

Los espacios virtuales de aprendizaje favorecen aspectos que la presencialidad limita o simplemente no contempla. Entre estas facilidades de la virtualidad, las más relevantes se asocian con el rompimiento de la barrera de la distancia, la rigidez de los horarios y la facilidad de la distribución del tiempo de estudio. (Moreira-Segura y DelgadilloEspinoza, 2015, p.2) 
Es por esta razón que las herramientas tecnológicas son un mediador de suma importancia, en la educación, por el mismo hecho de que brinda información variada y es accesible, contribuye también al manejo adecuado y flexible de la distribución de tiempo que cada individuo tenga a disposición. la virtualidad es un concepto que, sin ser lo mismo, va asociado a este impacto tecnológico en el mundo educativo. Por lo tanto, la virtualidad es una nueva manera de relación, de igual forma un espacio de comunicación atemporal, asíncrona o síncrona, brinda la posibilidad de construir entornos nuevos de interacción sin la necesidad de compartir el espacio-tiempo (Zambrano, Laurencio y Milán, 2018).

\section{Características de la virtualidad}

La virtualidad, comprende de una serie de conjuntos técnicos que satisfacen las necesidades del individuo en su aspecto natural como investigador, de acuerdo con Duran (2015) la virtualidad es útil como ámbito de información, construcción, y creación de experiencias de aprendizaje y descubrimiento. Por esta razón es una herramienta importante en la educación, no solamente como un recurso didáctico, también como un medio de comunicación que favorece al desarrollo de habilidades y capacidades en el individuo.

Por otro lado, Carbero (1998) alude algunas características de la virtualidad que se sintetizan en los siguientes puntos: a) Información multimedia: es el proceso de transmisión de la información, imagen, textual y de sonido, los avances tecnológicos han permitido mejorar la calidad de los procesos virtuales; b) La interactividad: es una de las características virtuales más relevantes, sobre todo en el ámbito educativo, por medio de la virtualidad se puede intercambiar información entre usuario a usuario, o usuario a ordenador, está característica admite adaptar los recursos a las múltiples necesidades de cada usuario; c) La interconexión: se refiere a la creación de posibilidades tecnológicas nuevas de la virtualidad, partiendo de la conexión entre dos tecnologías, por ejemplo, la virtualidad y las Tics.

También se abordan temas relacionados con las oportunidades espacio-temporales de la virtualidad: a) La inmaterialidad: hace referencia a el proceso y la comunicación de la información, esta información es fundamentalmente inmaterial y puede ser llevada de forma transparente e instantánea a lugares que son lejanos. b) La instantaneidad: la virtualidad y su integración informática han posibilitado, la comunicación y la transmisión de la información tenga un alcance completo, en el sentido que llega a cualquier lugar y de manera rápida.

c) Tendencia a la automatización: las características que posee la tecnología empujan a la aparición de diversas posibilidades y herramientas que permiten un manejo automático de la información, en diversos ámbitos; profesionales, personales y sociales.

\section{Importancia de la virtualidad}

Hoy en día la virtualidad está inmersa en todas las esferas; sociales, económicas y culturales, y por este motivo es evidente la contribución que otorga en cada espacio, dado esto es posible mencionar que la virtualidad es importante porque, ofrece un abanico de beneficios como; ayuda a organizar el tiempo, es facilitador de la información, la comunicación, posibilita la educación de manera amplia, estimula el conocimiento y la investigación, según Sangrá (2001) menciona que: 
Educación y virtualidad se complementan en la medida en que la educación puede gozar de las posibilidades de creatividad de la virtualidad para mejorar o diversificar sus procesos y acciones encaminados a la enseñanza y al aprendizaje, mientras que la virtualidad como sistema se beneficia de la metodología de trabajo educativo y de comunicación, necesaria en aquellos casos habituales en los que la finalidad de la relación en la red sobrepasa la de la búsqueda de información. (p. 119)

En relación con lo citado, se destaca que los diversos recursos que ofrece la tecnología y la virtualidad misma son relevantes para el proceso de enseñanza-aprendizaje, posibilitando que el estudiante y el educador gocen de los beneficios que caracteriza a la virtualidad como un sistema completo.

\section{Definición de las prácticas preprofesionales}

Es una modalidad que permite poner en prácticas la teórica dada en el salón de clases, con el fin de obtener una óptica más clara del campo de trabajo, según Chávez, Chancay, Del Pilar y Mendoza (2019) las prácticas son "un conjunto de acciones que realiza el practicante dentro del aula con fines educativos, estas acciones llevan implícitos conocimientos y creencias que determinan el desarrollo de saberes y posibilidades que le permiten desarrollar su trabajo" (p.6). De esta manera los practicantes aplican sus conocimientos, habilidades, destrezas y aptitudes adquiridas previamente en las entidades educativas, aportando de esta forma al desempeño en el campo laboral.

Las prácticas son el primer acercamiento a la vida laboral, por ello se requiere que el alumno palpe la realidad contextualizada de lo que supone un trabajo, Por otro lado, desde la perspectiva de Gajardo (2016) "Son muchas las oportunidades de crecimiento que ofrecen las prácticas, es por esto es totalmente fundamental saber si son relevantes o no, y sobre todo saber si influyen en el futuro desempeño profesional" (p.5). Considerando que es un periodo donde los alunamos se vinculan directamente con las obligaciones y responsabilidades que deben ejecutar en su futura vida profesional, se destaca que es un aspecto indispensable.

\section{Importancia de las prácticas preprofesionales}

Las prácticas preprofesionales se han convertido en un requisito indispensable para los estudiantes de las diferentes carreras, en las diferentes modalidades, en notable los cambios que ha tenido la sociedad y las necesidades que han surgido por esta razón la formación profesional debe mejorar en virtud de lo que requieren los avances, la práctica "contribuye a la adquisición de conocimientos y el desarrollo de competencias, así como un vínculo afectivo con la profesión. Lo cual permite la reafirmación de los intereses profesionales, así como el desarrollo de los modos de actuación profesional" (Rojas, Estévez, y Domínguez, 2017, p.45).

La pertinencia en las prácticas pre profesionales, no solo radica en que es requisito previo a la obtención de un título, sino que es una modalidad de formación que permite al estudiante desarrollar competencias laborales, lo que posiciona al futuro profesional a obtener un puesto de trabajo en su área y sus beneficios. 
El Ministerio de Educación del Ecuador (2016) señala que las prácticas brindan un entorno favorable donde el practicante debe de manifestar diferentes aspectos como los siguientes: a) Cumplir con el plan de actividades entregado por el docente tutor académico y con las actividades establecidas por el docente guía o tutor profesional para la planificación pedagógica; b) Mantener una actitud de colaboración y de equipo con los miembros de la comunidad educativa con los que interactúa; c) Conservar la confidencialidad de la información propia de la institución educativa donde realiza la práctica; d) Informarse sobre los lineamientos y políticas que rigen las acciones de la IE a la que ha sido asignado.

En consecuencia, a esto, las prácticas están inmersas en el proceso de titulación de los estudiantes, como uno de los aspectos más destacados de la formación como profesional, esta modalidad permite al estudiante poner de manifiesto los saberes adquiridos y transformarlos en competencias.

\section{Generalidades de las prácticas preprofesionales}

Los procesos de prácticas preprofesionales requieren el desarrollo de planes que consideren varios aspectos para su organización, entre ellos: los espacios propios de cada carrera, la cantidad de horas que debe ejercer el estudiante y los convenios que hay por parte de las entidades educativas con los diferentes organismos. En el artículo 9 del reglamento de prácticas pre profesionales impartido por el Consejo de Educación Superior (CES) (2017) menciona que:

Las prácticas pre profesionales deben responder a un programa prestablecido debidamente monitoreado y evaluado, el mismo que debe ser elaborado anualmente de manera institucional o de acuerdo a los periodos definidos en cada Facultad según sea la situación académica de los estudiantes a fin de que estos programas permitan la aplicación práctica del conocimiento, habilidades, destrezas y actitudes adquiridas por el estudiante durante su proceso de formación de acuerdo a las exigencias del mercador ocupacional.(p. 6)

Del mismo en el reglamento de prácticas pre profesionales dado por el CES (2017), se abordan los procedimientos para la planificación de las prácticas pre profesional, los cuales son:

1. La dirección de relaciones interinstitucionales enlistará y enviará la documentación de los estudiantes que se encuentren en condiciones de acceder a este proceso en cada semestre a fin de que puedan ejecutar las prácticas o las pasantías para el seguimiento y cumplimiento de los procesos de prácticas pre profesional.

2. Los coordinadores asignados de prácticas de carrera juntamente con los tutores académicos deben preparar un programa de prácticas pre profesionales a desarrollar. Dentro de las condiciones estipuladas durante los procesos formativos está que se debe cumplir la realización de prácticas, que han establecido la facultad o la carrera.

3. En concordancia con las listas de los estudiantes que están en condiciones de acceder a los procesos de realización de prácticas y en función de cada especialización de los estudiantes de las diversas carreras, sé designara la institución pública o privada, en la que el estudiante realizara sus prácticas, en caso de que el estudiante por sus propios medios ubique una institución ya sea 
pública o privada para realizar este proceso, será parte de una formalización de las prácticas pre profesionales.

4. Para la formalización de las prácticas pre profesionales, el coordinador de prácticas de carrera elaborará y enviará a las instituciones públicas o privadas una carta de solicitud para la realización de este proceso en función del reglamento;

5. Cada coordinador de prácticas deberá realizar un seguimiento, para obtener por escrito la respuesta positiva a la solicitud de las prácticas pre profesionales.

6. Obtenidas las respectivas aceptaciones por parte de cada institución se debe formalizar con la firma de un convenio de colaboración entre las instituciones públicas o privadas y la institución de Educación Superior.

7. Notificar a los estudiantes los datos y requisitos necesarios que permitan efectuar el contacto pertinente y por consiguiente el inicio de prácticas.

8. El coordinador de prácticas por carrera deberá dar seguimiento al desarrollo de prácticas pre profesionales, para la realización de fichas y anexos correspondientes.

Cabe señalar que el proceso de prácticas pre profesionales se adaptó a la realidad circundante, poniendo de manifiesto la puesta en práctica de los conocimientos adquiridos de los estudiantes a través del uso de herramientas virtuales. Es por ello por lo que para continuar con el proceso de enseñanza aprendizaje, el Instituto Superior Tecnológico Tsáchilas (2020) estableció un cronograma de actividades para las prácticas virtuales de formación dual durante la emergencia sanitaria, que consta de las siguientes directrices:

1. Designación de los estudiantes, por parte del responsable de prácticas de formación. Dual, de la carrera de Desarrollo Infantil Integral.

2. Capacitación virtual para la Modalidad Dual, sobre el Plan Marco de Formación por parte de los tutores empresariales a estudiantes.

3. Socialización del repositorio digital para la organización de fichas, en la ficha 2, el estudiante deberá llenar con información personal y con información correspondiente de la institución de convenio, adjuntando anexos solicitados como: fotos de la institución y dirección de Google Maps.

4. El tutor empresarial responsable, elaborará un cronograma de visitas de forma virtual, para ello cada estudiante compartirá su link de videoconferencia, a su tutor, para el monitoreo correspondiente de la ejecución de la clase.

5. Cada tutor empresarial llevara un registro de las visitas realizadas, en una matriz de Excel, bajo los siguientes criterios: Nombre de él o la estudiante, día, hora de inicio de finalización, datos institucionales, nombres de los tutores empresariales, correos, números de contacto, evidencia de la reunión a través de anexos fotográficos. 
6. Después del seguimiento el docente tutor asignado a la visita y el estudiante, completarán, la ficha 3 de supervisión de prácticas, en esta ficha se registrarán los procesos que se hicieron durante la visita, también se añade los siguientes criterios: fecha, hora, dificultades y fortalezas de la actividad práctica, asimismo se añadirán sugerencias y soluciones, esta ficha se anexará en el repositorio digital antes ya explicado.

7. El tutor académico además recogerá las siguientes fichas virtuales: a) Ficha 4 de evaluación del tutor, con firma digital y certificado en pdf; b) Ficha 5 de evaluación del Proyecto Integrador de Saberes (PIS); c) Ficha 6 de registro de actividades, dentro de esta ficha se observan las actividades llevadas a cabo por los estudiantes durante la práctica virtual a fin de que estas respondan al plan marco, misma que debe contener fotografías, links u otros tipos de anexos que sirven para evidenciar las actividades; d) Ficha 7; corresponde al plan marco de formación, el tutor empresarial mantendrá actualizado el drive de prácticas de la carrera, el portafolio de prácticas, en formatos pdf, con su respectivo checklist.

8. Se entregará a coordinación de la carrera las notas de la fase práctica de primer parcial y segundo parcial, para registrar en el sistema.

9. Se entregará al responsable de la carrera, las bitácoras de los PIS, con enlace de los videos de exposición, actas y rubricas, así como también los productos técnicos generados en el proceso de formación dual.

10. Se registrará el cumplimiento de los estudiantes en las prácticas virtuales.

11. Se entregará los certificados de prácticas pre profesionales.

Los puntos antes enlistados se establecieron con el afán de organizar los subprocesos de la práctica pre profesional delimitando las actividades de tutores empresariales, estudiantes y tutores responsables de la práctica pre profesional. En este sentido, se proyectó a aprovechar las potencialidades de la virtualidad para organizar todo el proceso de formación dual. Es importante el cronograma de actividades delimitado, ya que definía cada uno de los procesos que debían seguir los implicados de forma sistemática y secuencial, además, se establecieron las fichas de evidencia a presentar por estudiantes y sus formas de completamiento, rúbricas y documentos a llenar por los tutores empresariales.

\section{Metodología}

El enfoque utilizado para el desarrollo de la investigación fue el cualitativo, dado que se pretende describir y detallar teóricamente situaciones determinadas, que son observables para el sujeto. Según Hernández, Fernández y Baptista (2014):

El enfoque cualitativo puede concebirse como un conjunto de prácticas interpretativas que hacen al mundo "visible", lo transforman y convierten en una serie de representaciones en forma de observaciones, anotaciones, grabaciones y documentos. Es naturalista (porque estudia los fenómenos y seres vivos en sus contextos o ambientes naturales en su cotidianidad) e interpretativo (pues intenta 
encontrar sentido a los fenómenos en función de los significados que las personas les otorguen). (p.42)

De igual manera, el estudio es de tipo de descriptivo, porque se requerirá especificar y puntualizar las características de determinado fenómeno, que esté sujeto a estudio y valoración (Tamayo, 2012).

La población de estudio está compuesta por 347 individuos que conforman la totalidad personas de la carrera de Desarrollo Infantil Integral del Instituto Superior Tecnológico Tsáchila. A través de un muestreo, no probabilístico por conveniencia, se seleccionó a 45 estudiantes del quinto A y B como muestra, teniendo en cuenta los siguientes criterios de selección: dificultades recursivas, accesibilidad recursiva, ubicación geográfica y madres o padres de familia. De igual manera, se eligió a dos docentes (coordinadora de carrera y de prácticas preprofesionales), a fin de contrastar las perspectivas, discentes y docentes.

Las técnicas que se utilizaron para la recolección de datos, en el presente estudio, fueron:

a) Grupo focal: de acuerdo con Edouard (2017) afirma que el grupo focal "es un tipo de entrevista en grupo a personas beneficiadas o afectadas por una política pública o por la intervención de un proyecto y/o programa. Es una técnica diseñada para obtener información acerca de las percepciones, actitudes, experiencias y expectativas en relación a una política, proyecto y/o programa" (p.3).

b) Entrevista: es un intercambio interactivo de información, que se lo emplea en forma de diálogo y permite al entrevistador conocer de forma más amplia lo que precisa. De acuerdo con Díaz, García, Martínez y Valera (2013) la entrevista es "una técnica de gran utilidad en la investigación cualitativa, para recabar datos" (p.2).

Como instrumentos de recolección de datos, en primera instancia para la técnica del grupo focal se utilizó: cuestionario semiestructurado, este se caracteriza por poseer preguntas abiertas y deja espacio al investigador para continuar indagando a través de una conversación estructurada que tiene como guía las preguntas iniciales orientadas a un tema en específico.

Del mismo modo, para la consecución de la entrevista, basados en las definiciones de Hamui y Varela (2012) se utilizó como instrumento: entrevista semiestructurada, que se trata de una reunión de dos personas para abordar una temática específica, en este caso, de forma semiestructurada, es decir, con preguntas abiertas que guían una conversación heurística. Ambos instrumentos cuentan con una valoración por criterio de expertos, lo que garantiza la pertinencia y coherencia de las preguntas. Cabe recalcar que, para la recolección de datos, se realizaron solicitudes a las máximas autoridades académicas para obtener el consentimiento informado de los implicados en la investigación.

Para el análisis de los datos se utilizó a la técnica de reducción y categorización propuesta por Huberman y Miles (1994) que ofrece una mirada crítica para construir una teorización conclusiva. Es decir, a partir de las interpretaciones individuales de la problemática, el investigador realiza una síntesis interpretativa del fenómeno. Esta técnica de análisis de datos se compone de tres subprocesos: a) La reducción de datos: en esta etapa se realiza una selección de los criterios y 
se los condensa a través de resúmenes; b) Presentación de datos: se agrupa la información en organizadores que facilitan la reflexión de los criterios concretados en las presentaciones; c) Elaboración de síntesis: se realizan conclusiones que permiten la extracción de los resultados a través de la comparación de criterios y contraste de ideas en función de patrones determinados.

\section{Resultados}

\section{Resultados de los grupos focales:}

Las características problemáticas para la selección de los grupos focales y su organización se pueden observar a continuación en la tabla 1.

\section{Tabla 1}

Criterios para la organización de grupos focales

\begin{tabular}{cccc}
\hline Grupos & Criterio de selección & Número de participantes & Género \\
\hline Grupo 1 & Dificultades recursivas & 5 & Femenino \\
Grupo 2 & Dificultades recursivas & 5 & Femenino \\
Grupo 3 & Accesibilidad recursiva & 5 & Femenino \\
Grupo 4 & Ubicación geográfica & 5 & Femenino \\
Grupo 5 & Ubicación geográfica & 5 & Femenino \\
Grupo 6 & Madres o padres de familia & 5 & Femenino \\
Grupo 7 & Madres o padres de familia & 5 & Femenino \\
Grupo 8 & Madres o padres de familia & 5 & Femenino \\
Grupo 9 & Madres o padres de familia & 5 & Femenino \\
\hline
\end{tabular}

Asimismo, en la tabla 2 se considera oportuno detallar las dimensiones (D) e indicadores (I) evaluados en la investigación, que se encuentran relacionados con los subprocesos establecidos en el cronograma de procesos para las prácticas duales durante la emergencia sanitaria elaborado por el Instituto Superior Tecnológico Tsáchila (2020):

\section{Tabla 2}

Dimensiones e indicadores evaluados en la investigación

\section{Dimensiones \\ Indicadores}

D1.I1. Asignación de los estudiantes/tutores.

D1.I2. Capacitación virtual/Plan marco de formación.

D1. Fase de planificación de la D1.I3. Socialización de repositorio digital para la organización de práctica preprofesional. documentos de la práctica.

D1.I4. Cronograma de prácticas pre profesionales.

D2. Fase de desarrollo de la práctica preprofesional.
D2. I1. Fase de observación y diagnóstico.

D2. I2. Fase de planificación. 
D2. I3. Fase de práctica pedagógica.

D2. I4. Seguimiento por parte del docente tutor asignado a la visita.

D2. I5. Fase de informe final y presentación de experiencias.

D3. Fase de evaluación de la práctica preprofesional.
D3. I1. Evidencia y dificultades en la práctica.

D3. I2. Rendimiento pre profesional de los estudiantes.

A continuación, se presenta una síntesis de los resultados obtenidos entre los nueve grupos focales aplicados.

D1. Fase de planificación de la práctica preprofesional:

D1.I1. Asignación de los estudiantes/tutores.

Pregunta: ¿Qué tipo de influencia tuvo la virtualidad para la asignación de los estudiantes y tutores para la práctica pre profesional?

Los estudiantes de los nueve grupos focales manifestaron que el impacto fue negativo, porque en las asignaciones para la práctica preprofesional no se consideraron las realidades económicas de cada uno de ellos. Algunos de los estudiantes no contaban con recursos tecnológicos y didácticos para llevar a cabo una clase virtual. Asimismo, no se brindaron actividades alternas para los estudiantes que tuvieron dificultades para desarrollar las prácticas de manera virtual, debido que algunos de los estudiantes no contaban con los recursos, tecnológicos y didácticos para llevar a cabo una clase virtual. Recalcan que la institución fue inflexible al momento de asignar, mencionan que se aplicó una encuesta para ver dificultades en los estudiantes, sin embargo, no se tomó ninguna medida al respecto.

\section{D1.I2. Capacitación virtual/Plan marco de formación.}

Pregunta: ¿Qué tipo de influencia tuvo la virtualidad para el proceso inicial de capacitación de las prácticas pre profesionales y la socialización del plan marco de formación?

Los estudiantes recalcan que, los procesos iniciales de capacitación tuvieron un impacto negativo, porque no fueron de calidad, ni suficientes para desarrollar la práctica de manera virtual, dado que esta modalidad es nueva, por lo tanto, consideraron que las capacitaciones que se dieron no abordaron todos los recursos virtuales que se podrían utilizar para el desarrollo de la práctica y los contenidos tratados no fueron de calidad. En cuanto a la socialización de plan marco de formación, los estudiantes concuerdan en que este fue un proceso que no se comprendió, ya que el proceso de su socialización quedó inconcluso. práctica.

D1.I3. Socialización de repositorio digital para la organización de documentos de la 
Pregunta: ¿Qué tipo de influencia tuvo la virtualidad en la creación y socialización del repositorio digital para la organización de documentos de la práctica pre profesional?

Los estudiantes de todos los grupos focales concuerdan en que este proceso tuvo, por un lado, un impacto negativo, puesto que no se dieron las directrices pertinentes para completar información de cada ficha, únicamente se socializó la creación y organización de carpetas digitales. Por otro lado, también tuvo una influencia positiva, reconociendo que les brindo una mayor facilidad, ya que hubo ahorro de gastos porque todos los documentos se organizaron de forma digital en la plataforma, esto también supone una ventaja al momento de hacer las correcciones.

\section{D1.I4. Cronograma de prácticas pre profesionales. \\ Pregunta: ¿Qué tipo de influencia tuvo la virtualidad para la realización y socialización del cronograma de prácticas pre profesionales?}

En la mayor parte de grupos focales, los estudiantes manifestaron que, este proceso tuvo un impacto negativo, porque no se tomó en cuenta la disponibilidad de cada uno de ellos para el desarrollo del cronograma de actividades, también conceptuaron que este proceso fue incomprensible. En contraposición, en algunos grupos focales se mencionó que en este indicador la virtualidad tuvo un impacto positivo, ya que facilitó la realización y socialización del cronograma con rapidez, mediante comunicación sincrónica.

Los resultados obtenidos desde la fase de planificación reflejan una problemática latente, en primera instancia en términos recursivos, que puede tener su origen en las características socioeconómicas de los estudiantes del Instituto Superior Tecnológico Tsáchilas. Sin embargo, en una carrera tecnológica, que tiene como punto esencial y permanente la formación dual, es indispensable la asignación, planificación, organización, dirección, aplicación y control de las prácticas; no obstante, también era necesario elaborar planes de contingencia para solventar la imposibilidad tecnológica, geográfica o recursiva que imposibilite a los estudiantes el desarrollo de su formación dual. Estos resultados se relacionan con lo expuesto por la UNESCO (2020) sobre que más del 91,3\% de estudiantes han sido afectados por el cierre de instituciones, ya que carecen de recursos tecnológicos, por ende, a partir de estos hallazgos, se podría deducir que la educación superior, no fue la excepción.

Por otro lado, también existen dificultades en relación a las instrucciones brindadas mediante la virtualidad para el desarrollo de la práctica pre profesional. Los estudiantes coincidieron, en su mayoría, en que no fueron suficientes ni de calidad, además, no abordaron todos los contenidos. Quizá esto pueda relacionarse también con las dificultades recursivas de los estudiantes o en su lugar, a la adaptación repentina de los docentes a herramientas de comunicación virtual, ya que no hubo tiempo para una preparación más detallada. Esto coincide con lo mencionado por Zambrano, Laurencio y Milán (2018) donde se analiza que la virtualidad es una nueva manera de relación, brinda posibilidades, pero requiere de preparación detallada para organizar de manera pertinente la comunicación. También, se debe tener en cuenta que los estudiantes se encontraban acostumbrados a recibir este tipo de capacitaciones de manera presencial. 
Sin embargo, el desarrollo de esta fase desde la virtualidad también tuvo algunos aspectos positivos que recalcan los estudiantes, como es el caso del ahorro económico que tuvieron, ya que no tenían que imprimir fichas de prácticas, movilizarse para hacerlas firmar o dirigirse a las instituciones a realizar la práctica pre profesional, esto permite inferir que la virtualidad tiene potencialidades por explorar y que mediante una planificación oportuna, con mecanismos de contingencia para atender todas las casuísticas podría resultar productiva para las carreras de formación dual. Ya hablaba de ello Sangrá (2001) a inicios de la década, donde comentó que la educación y virtualidad se complementan de modo en que sobrepasa una relación única de búsqueda y almacenamiento de información, llegando a mejorar y diversificar los procesos educativos. Pero, como mencionan García, Guerrero y Granados (2015) para que los estudiantes se sientan cómodos trabajando en los EVEA se necesitan buenas prácticas profesionales.

\section{D2. Fase de desarrollo de la práctica preprofesional. Indicadores:}

D2. I1. Fase de observación y diagnóstico.

Pregunta: ¿Qué tipo de influencia tuvo la virtualidad en la fase de observación y diagnóstico de la comunidad educativa que se realiza previamente al desarrollo de planificaciones a aplicar en la práctica pre profesional docente?

Los grupos focales coinciden en que esta fase tuvo un impacto negativo, porque la virtualidad limitó observar las dificultades que podían presentarse, sin embargo, otro grupo expone que, la virtualidad tuvo un impacto positivo, porque facilita la observación de características sociofamiliares importantes para el diagnóstico y la elaboración de los planes de clase. Sin embargo, fueron mayores los problemas identificados en los estudiantes, quienes mencionaban que el diagnóstico no se desarrollaba en un clima de calidez, con ambientes de confianza por el entorno socio-familiar regular y malo de algunas familias.

\section{D2. I2. Fase de planificación.}

Pregunta: ¿Qué tipo de influencia tuvo la virtualidad en la fase de planificación para impartir clases en el desarrollo de la práctica pre profesional?

La mayor parte de estudiantes manifestaron que, la virtualidad en esta fase tuvo un impacto negativo, porque no tenían el conocimiento suficiente para planificar clases virtuales y no tenían un apoyo docente para hacerlo. Del mismo modo comentaron que no contaban con los materiales o desconocían el uso de programas y recursos virtuales idóneos.

\section{D2. I3. Fase de prática pedagógica.}

Pregunta: ¿Qué tipo de influencia tuvo la virtualidad en la fase práctica pedagógica, donde se desarrollaban los procesos de estimulación concernientes a las prácticas pre profesionales?

En los grupos focales se encontraron criterios como: que este proceso tuvo un impacto negativo, porque no tuvieron disponibilidad ni destreza en el uso de las TIC, para el desarrollo de sus clases, y que, a percepción de ellos como estudiantes, falto mucho para una clase virtual de calidad, resaltan que intentaron dar dentro de sus posibilidades una clase virtual enriquecedora, pero que se limitaron mucho por el desconocimiento. De igual manera, se menciona que tuvo un 
impacto positivo, porque adquirieron experiencias para dar clases de manera virtual, lo que hoy en día supone un gran reto para la realidad contemporánea.

D2. I4. Seguimiento por parte del docente tutor asignado a la visita.

Pregunta: ¿Qué tipo de influencia tuvo la virtualidad en el seguimiento de la práctica pre profesional por parte de los docentes tutores?

En este indicador todos los estudiantes concuerdan en que la influencia fue positiva, ya que los docentes dieron seguimiento de manera estricta a cada uno de los practicantes, enlazándose a las clases en vivo para presenciar las actividades de estimulación y evaluar el desempeño de todos.

D2. I5. Fase de informe final y presentación de experiencias.

Pregunta: ¿Qué tipo de influencia tuvo la virtualidad en la fase del desarrollo del informe final y presentación de experiencias de la práctica pre profesional?

En todos los grupos, los estudiantes enuncian que, el impacto que tuvo esta fase fue positivo, porque la virtualidad facilitó el desarrollo del proceso de realización de informes y documentos finales. Representó ahorro, ya que las correcciones y errores se las subsanaban en línea. Además, se podía tener un control constante de los documentos a elaborar y los que se encontraban pendientes, debido que todas las carpetas se encontraban en un mismo lugar en drive y era posible tomar de ejemplo a otro compañero.

En la fase de desarrollo los resultados permiten observar mayormente un impacto negativo de la virtualidad, puesto que, en primera instancia se limitó el diagnóstico de las dificultades que presentaban los estudiantes previos al inicio de las actividades de estimulación. Esto concuerda con lo que planteaba la UNICEF (2020) referente a que la crisis de formación humana se agravaría con la emergencia sanitaria. De igual manera, también existen otras dificultades relacionadas con diversos ámbitos, como es el caso de la carencia de conocimientos que presentaban los estudiantes para planificar de forma autónoma, la ausencia de recursos para adecuar entornos de estimulación en sus hogares y la falta de dominio de programas para el desarrollo de clases virtuales. Por estas dificultades mencionadas Guarnizo (2018) comentaba que para la realización de prácticas pre profesionales en entornos virtuales era sustancial elaborar un proceso de formación sistemático que adapte a los estudiantes al nuevo entorno laboral.

No obstante, también existieron unos pocos aspectos positivos, como es el caso de la posibilidad de adentrarse como estudiantes a las características socio-familiares que brinda una óptica de la situación, limitaciones y oportunidades del infante. De igual manera, los informes finales no representaron gastos económicos excesivos como en otras ocasiones. Esto permite inferir que, la virtualidad debería complementar a la práctica pre profesional en la presencialidad, evitando gastos dispensables y potenciando procesos que resultan más sencillos y operativos en ambientes virtuales.

D3. Fase de evaluación de la práctica preprofesional.

D3. I1. Evidencia y dificultades en la práctica.

Pregunta: ¿Qué tipo de influencia tuvo la virtualidad para la identificación de dificultades y la recopilación de evidencia de la práctica pre profesional? 
En los grupos focales esta pregunta presenta mayor divergencia, ya que, existen varios donde se menciona que la virtualidad tuvo un impacto negativo, porque no se contó con la colaboración completa de los padres para la recopilación de evidencias de actividades. Además, la mala conexión a internet y la limitada visualización del espacio dificultaba la percepción de dificultades. Por otro lado, en una menor cantidad de grupos focales se tuvo en cuenta que el impacto de este proceso fue positivo, ya que al asumir directamente un rol de educador y ya no de acompañante como lo suelen hacer en las prácticas recurrentes, les permitió evidenciar algunas dificultades y la recopilación de evidencias de las mismas a través de las posibilidades que ofrecen las TIC.

\section{D3. I2. Rendimiento pre profesional de los estudiantes.}

Pregunta: ¿Qué tipo de influencia tuvo la virtualidad en el rendimiento de los estudiantes en la práctica pre profesional?

En todos los grupos focales se establece que el impacto de la virtualidad en el rendimiento académico de los estudiantes de la práctica preprofesional fue positivo ya que todos obtuvieron calificaciones máximas. Se recalca también que, en anteriores periodos, el rendimiento en la práctica preprofesional no era tan alto, sin embargo, a pesar de las dificultades consiguieron notas perfectas.

En la fase de evaluación, los resultados demuestran perspectivas variadas. Por un lado, algunas personas consideran que la práctica pre profesional tuvo un impacto positivo puesto que, contaban con mayor independencia para impartir clases y consiguieron notas perfectas. Esto se encuentra relacionado con lo que menciona Knowles (2006) acerca de que los adultos tienen una profunda necesidad de autodirigir sus procesos de aprendizaje. Por ende, se podría decir que, en la práctica pre profesional se debe brindar mayor autonomía al estudiante, sin descuidar la preparación necesaria requerida y la consideración de sus posibilidades económicas.

Otro punto a comentar que resulta curioso, son las calificaciones obtenidas, que, a diferencia de otros periodos, fueron perfectas, pero no se encuentran relacionadas al rendimiento y la satisfacción de los estudiantes. Por ello, se podría decir que, no se evaluó de forma correcta el desempeño profesional de la práctica pre profesional en ambientes virtuales. Se recomienda revisar los criterios de evaluación y tener en cuenta un análisis de las competencias que permita observar el desarrollo de conocimientos conceptuales, procedimentales y actitudinales puestos en práctica.

Por otro lado, continúan las perspectivas de impacto negativo de la virtualidad en el desarrollo de la práctica, donde las dificultades de conectividad y la falta de recursos familiares afectaron la recolección de evidencias para la elaboración de informes finales. Esto refleja que es importante un análisis de las condiciones socioeconómicas del contexto previo a la incorporación de la virtualidad en la práctica pre profesional. Se requiere un análisis minucioso y planificaciones sistemáticas cuidadosas para no presentar este tipo de problemas.

\section{Resultados de entrevistas a docentes:}


De igual manera, se tuvo en cuenta los mismos indicadores consultados a los estudiantes para realizar las entrevistas a docentes. A continuación, se establecen las respuestas del docente coordinador de la carrera (DCC) y del docente coordinador de prácticas preprofesionales (DCPP).

D1. Fase de planificación de la práctica preprofesional.

D1.I1. Asignación de los estudiantes/tutores.

Pregunta: ¿Qué tipo de influencia tuvo la virtualidad para la asignación de los estudiantes y tutores para la práctica pre profesional?

DCPP: la influencia de la virtualidad durante este proceso fue positiva, se agilizó la distribución del alumnado a la entidad responsable, también se puede observar que benefició al estudiante al ya no tener que movilizarse lejos, esto porque a virtualidad es flexible en espacio y tiempo.

DCC: el proceso tuvo aspectos positivos y negativos. Los aspectos positivos fueron que los estudiantes continuaron con este proceso de prácticas y que por consiguiente no se atrasaron, Además la modalidad virtual fue más flexible. Por otro lado, los aspectos negativos fueron que algunos estudiantes presentaron dificultades para el desarrollo de prácticas porque no contaban con disponibilidad, conectividad, recursos didácticos, y espacios idóneos para la ejecución de los servicios de estimulación temprana.

\section{D1.I2. Capacitación virtual/Plan marco de formación.}

Pregunta: ¿Qué tipo de influencia tuvo la virtualidad para el proceso inicial de capacitación de las prácticas pre profesionales y la socialización del plan marco de formación?

DCPP: la influencia de la virtualidad fue positiva porque durante el desarrollo de tutorías y capacitaciones, asistieron en su gran mayoría los estudiantes, tutores empresariales y otros involucrados, pese a que un porcentaje mínimo de estudiantes no logro acceder a estos procesos, se subsanó con tutorías.

DCC: En este punto, tuvo un impacto negativo, porque las capacitaciones y socializaciones acerca del plan marco de formación, como de otros documentos, no tuvieron un tiempo estimado idóneo, por ello, este aspecto supuso una dificultad porque los estudiantes no pudieron adquirir información para el correcto manejo de los procesos de prácticas de manera virtual. práctica.

D1.I3. Socialización de repositorio digital para la organización de documentos de la

Pregunta: ¿Qué tipo de influencia tuvo la virtualidad en la creación y socialización del repositorio digital para la organización de documentos de la práctica pre profesional?

DCPP: la influencia de la virtualidad fue positiva, porque facilito los procesos de organización documental de la práctica pre profesional, distribuyendo dicha documentación en un repositorio digital de acceso compartido.

DCC: este proceso tuvo un impacto positivo, porque facilito tanto a estudiantes como a docentes la creación de documentos y la organización de los mismos para cumplir con los requisitos de prácticas. 
D1.I4. Cronograma de prácticas pre profesionales.

Pregunta: ¿Qué tipo de influencia tuvo la virtualidad para la realización y socialización del cronograma de prácticas pre profesionales?

DCPP: este proceso tuvo una influencia positiva, porque se acaparo información puntual sobre la realización del cronograma de prácticas, y luego se socializó con los practicantes de manera sincrónica.

DCC: tuvo un impacto positivo, porque se hizo de manera entendible y óptima. Los cronogramas ayudaron a los estudiantes a organizarse de mejor manera para dar cumplimiento al requisito curricular que son las prácticas preprofesionales.

D2. Fase de desarrollo de la práctica preprofesional. Indicadores:

D2. I1. Fase de observación y diagnóstico.

Pregunta: ¿Qué tipo de influencia tuvo la virtualidad en la orientación de los estudiantes para la fase de observación y diagnóstico de la comunidad educativa previa al desarrollo de la práctica pre profesional?

DCPP: tuvo un impacto positivo, dado que los tutores orientaron y dieron determinadas directrices para la observación y diagnóstico de los alumnos asignados a los practicantes.

DCC: pienso que la virtualidad tuvo una influencia positiva, porque les permitió observar, analizar y diagnosticar a la comunidad educativa a la que se dirige por medio de fichas, planificaciones y documentos.

D2. I2. Fase de planificación.

Pregunta: ¿Qué tipo de influencia tuvo la virtualidad en la fase de planificación de planes de clase para el desarrollo de la práctica pre profesional?

DCPP: fue positiva dado que los docentes dieron a conocer las pautas para el desarrollo de la planificación, además, los estudiantes tenían ya conocimientos previos.

DCC: tuvo un impacto positivo, ya que, como estudiantes ellos contaban con conocimientos previos sobre la realización de planes de clase que les permita ejecutar actividades pedagógicas de manera autónoma.

D2. I3. Fase de práctica pedagógica.

Pregunta: ¿Qué tipo de influencia tuvo la virtualidad en la fase práctica pedagógica o aplicación de planes de clase en el desarrollo de la práctica pre profesional?

DCPP: la fase pedagógica tuvo un impacto positivo, porque esta experiencia del proceso de enseñanza-aprendizaje a nivel virtual ayuda al estudiante a adquirir y desarrollar habilidades y competencias, laborales y tecnológicas.

DCC: creo que tuvo un impacto negativo, dado que la virtualidad si bien ofrece diversas facilidades, también resulta ser complicada para quienes no tienen dominio tecnológico, algunos 
estudiantes no pudieron realizar las prácticas de manera efectiva por la limitación del manejo recursivo de la tecnología.

D2. I4. Seguimiento por parte del docente tutor asignado a la visita.

Pregunta: ¿Qué tipo de influencia tuvo la virtualidad en el seguimiento de la práctica pre profesional?

DCPP: tuvo un impacto positivo, porque los tutores empresariales estuvieron en constante seguimiento a los estudiantes, también se dieron las respectivas orientaciones para la mejora continua de este proceso a fin de obtener un buen desempeño.

DCC: este proceso tuvo un impacto positivo, ya que los tutores tuvieron una constante interacción y monitoreo a los estudiantes durante la ejecución de sus prácticas, para asegurar la calidad de estos procesos virtuales de enseñanza.

D2. I5. Fase de informe final y presentación de experiencias.

Pregunta: ¿Qué tipo de influencia tuvo la virtualidad en la fase del desarrollo del informe final y presentación de experiencias de la práctica pre profesional?

DCPP: tuvo un impacto positivo, puesto que la modalidad virtual brindó facilidades para la realización de los documentos y presentación de experiencias y evidencias.

DCC: tuvo un impacto positivo debido a que no se encontró ninguna dificultad, por otro lado, se resalta que esta fase fue cómoda porque se realizó un modelo estructurado para que los estudiantes ingresen sus datos a fin de optimizar tiempo.

\section{D3. Fase de evaluación de la práctica preprofesional.}

D3. I1. Evidencia y dificultades en la práctica.

Pregunta: ¿Qué tipo de influencia tuvo la virtualidad para la identificación de dificultades y la recopilación de evidencia de la práctica pre profesional?

DCPP: tuvo un impacto positivo, dado que la virtualidad ayudó a los estudiantes a identificar y recopilar evidencia de los procesos de prácticas. La tecnología presta un sin número de herramientas que facilitan los procesos curriculares.

DCC: este proceso tuvo un impacto positivo porque la virtualidad permitió constatar las dificultades y la recopilación de evidencia mediante el seguimiento continuo que se realizó.

D3. I2. Rendimiento pre profesional de los estudiantes.

Pregunta: ¿Qué tipo de influencia tuvo la virtualidad en el rendimiento de los estudiantes en la práctica pre profesional?

DCPP: supuso una influencia positiva, en virtud de que se constató que su rendimiento fue alto. 
DCC: tuvo un impacto positivo, porque los estudiantes obtuvieron calificaciones sobresalientes.

La entrevista realizada al docente coordinador de prácticas preprofesionales (DCPP) expone que el impacto de la virtualidad fue positivo en todos los indicadores, mencionó que como docente de la carrera la institución brindo todos los acompañamientos requeridos para que los estudiantes logren llevar a cabo esta nueva modalidad adaptada por la institución, también destaco que la virtualidad beneficio mucho en aspectos de agilización tanto de los procesos como del desarrollo de documentación, por último el docente constato las aseveraciones a través del indicador de rendimiento de la práctica pre profesional, indicando que el alumnado en su totalidad consiguió tener un rendimiento alto. Esto representa que desde la perspectiva docente los procesos que estuvieron inmersos en la práctica fueron adecuados.

Los resultados obtenidos del docente coordinador de la carrera (DCC) destacan que el impacto de la virtualidad tuvo dos enfoques: positivo y negativo. Señaló que, desde la perspectiva como docente de la carrera, los aspectos positivos se hallan los siguientes indicadores; socialización de repositorio digital para la organización de documentos de la práctica, cronograma de prácticas, observación y diagnóstico, planificación, seguimiento por parte del docente, informe final y presentación de experiencias evidencia y dificultades y rendimiento profesional de los estudiantes. Sin embargo, el docente también destaca que hubo procesos que no se realizaron con pertinencia, dentro de los aspectos negativos se hallan los siguientes; asignación de estudiantes, capacitación virtual/plan marco de formación y práctica pedagógica.

De igual manera, el DCC Expuso que el cumplimiento efectivo de estos indicadores se vio afectado porque algunos estudiantes presentaron problemas para dar cumplimiento esta fase, también mencionó que los estudiantes no tuvieron el tiempo necesario para adquirir competencias que les permitan desarrollar clases de forma virtual. Asimismo, mencionó que la virtualidad ofrece muchas facilidades y oportunidades, pero estas resultan ser complicadas para quienes no tienen dominio tecnológico.

\section{Conclusiones}

Se identificó que el impacto de la virtualidad en la práctica preprofesional fue negativo desde la perspectiva estudiantil porque se encontraron varias dificultades en común relacionadas con los siguientes indicadores: a) Asignación de estudiantes: los sujetos de muestra expusieron que tuvieron dificultades recursivas que nos les permitió llevar a cabo con pertinencia este proceso, b) Capacitación docente: se indicó que las capacitaciones para la planificación y el desarrollo de la práctica pre profesional dictadas por las autoridades fueron insuficiente, por el motivo que no hubo mucho tiempo para socializar y enseñar todos los contenidos que se requerían conocer c) Planificación: dentro de este indicador los sujetos de muestra mencionaron que no tenían conocimiento sobre planificación de clases virtuales, por lo que, se encontraban desorientados; d) Práctica pedagógica: los estudiantes manifestaron que no tenían suficientes conocimientos o dominios sobre cómo utilizar los programas de reuniones virtuales y aplicaciones referentes al campo educativo. 
Por otro lado, desde la perspectiva docente, se comentó que la virtualidad influyó de manera positiva en todos los indicadores, a) Asignación de tutores y estudiantes b) Capacitación virtual c) Socialización del repositorio digital para la organización de documentos d) Cronograma de prácticas pre profesionales e) Planificación f) Práctica pedagógica g) Seguimiento por parte del docente tutor asignado h) Presentación de experiencias i) Evidencia dificultades de la práctica j) Rendimiento profesional de los estudiantes. Del mismo modo acotan que, la institución cumplió con todos los procesos, utilizando las ventajas de la tecnología y la comunicación sincrónica, tales como; flexibilidad, interactividad e instantaneidad.

Esto permite inferir que, para futuros procesos de práctica preprofesional virtual, es necesario mantener una comunicación asertiva y responsiva constante entre los estudiantes practicantes y los docentes a cargo, ya que, pese a existir problemas, el docente responsable los desconoce y piensa que toda marcha con índices altos de calidad. De igual manera, los docentes tutores de prácticas preprofesionales al enterarse de conflictos o dificultades deben emprender estrategias de intervención inmediata, inclusive, lo ideal sería realizar medidas de contingencia que se anticipen a la práctica preprofesional, durante la práctica preprofesional y después de la práctica preprofesional.

\section{Referencias Bibliográficas}

Carbero, J. (1998). Impacto de las nuevas tecnologías de la información y la comunicación en las organizaciones educativas. Grupo Editorial Universitario. https://bit.ly/3oQkJY7

Chávez Loor, M. D., Chancay Cedeño, C. H., Chávez Loor, Y. P., y Mendoza Bravo, K. L. (2019). Las prácticas pre profesionales y su impacto social. Rehuso, 4(2), 129-136. https://bit.ly/3sm19Eh

Consejo de Educación Superior. (2017). Reglamento de prácticas pre profesionales [Página web]. https://bit.ly/3tEijNx

Cornejo Abarca, José. (2014). Prácticas profesionales durante la formación inicial docente: análisis y optimización de sus aportes a los que aprenden y a los que enseñan a aprender "a enseñar". Estudios pedagógicos (Valdivia), $40 \quad$ (Especial), 239256. https://doi.org/10.4067/S0718-07052014000200014

Delgado Zamora, R. (2019). El m-learning, las ventajas de la utilización de dispositivos móviles en el proceso autónomo de aprendizaje. Revista de Ciencias Humanísticas y Sociales, 1(35), 29-38. https://bit.ly/3oHmnes

Díaz Bravo, L., García Torruco, U., Martínez Hernández, M., y Varela Ruiz, M. (2013). La entrevista, recurso flexible y dinámico. Investigación en Educación Médica, 2(7), 162-167. https://bit.ly/393TItE

Duran Rodríguez, R. A. (2015). La Educación Virtual Universitaria como medio para mejorar las competencias genéricas y los aprendizajes a través de buenas prácticas [Tesis doctoral, Universitat Politécnica de Catalunya]. https://bit.ly/3yHTjsb

Edouard, F. (2017). Guía taller con grupos focales con beneficiarios directos. Organización de las Naciones Unidas para la Alimentación y la Agricultura [Página web]. https://bit.ly/3fEbhTr

Gajardo Piña, M. (2016). Prácticas Profesionales y su valor en el futuro desempeño laboral [Tesis de pregrado, Universidad de Chile]. https://bit.ly/3bQi77j 
García Martínez, A., Guerrero Proenza, R. S., y Granados Romero, J. M. (2015). Buenas prácticas en los entornos virtuales de enseñanza-aprendizaje. Revista cubana de educación superior, 1(3), 76-88. https://bit.ly/3cYi8pB

Guarnizo Crespo, S. F. (2018). Importancia de las prácticas pre profesionales para los estudiantes de Educación Superior en la Universidad de Guayaquil. INNOVA Research Journal, 3(8), 14-25. https://doi.org/10.33890/innova.v3.n8.2018.717

Hamui Sutton, A. y Varela Ruiz, M. (2013). La técnica de grupos focales Investigación. Educación Médica, 2(5), 55-60. https://bit.ly/31BkL4q

Hernández Sampieri, R., Fernández Collado, C, y Baptista Lucio, P. (2014). Metodología de la investigación. Mc Graw Hill.

Herrera Orozco, G. N. (2019). Evaluación del impacto de las prácticas pre profesionales en los graduados de la escuela de Ciencias de la Educación [Tesis de pregrado, Pontificia Universidad Católica del Ecuador]. https://bit.ly/3udS8Nz

Huberman, M. A., y Miles, M. B. (1994). Qualitative Data Analysis: A Sourcebook of New Methods. Educational Evaluation and Policy Analysis, 8(3), 329-331. https://doi.org/10.2307/1163741

Martínez Hernández, L. M., Leyva Arellano, M. E., Félix Arellano, F. L., Cecenas Torreno, P. E. y Ontiveros Hernández, V. C. (2014). Virtualidad, ciberespacio y comunidades virtuales. Red Durango de Investigadores Educativos. https://bit.ly/3vbHoR0

Ministerio de Educación del Ecuador. (2016). Manual para la implementación de prácticas preprofesionales [Página web]. https://bit.ly/38YGwpV

Moreira-Segura, C., y Delgadillo-Espinoza, B. (2015). La virtualidad en los procesos educativos: reflexiones teóricas sobre su implementación. Revista Tecnología en Marcha, 28(1), 121129. https://bit.ly/3vgrF3e

Rodríguez Andino, M. C., Calle García, R. X., y Zabala Espín, S. K. (2018). Modelo para desarrollar prácticas preprofesionales reflexivas en la formación de docentes para educación básica. Revista ESPACIOS, 39(46), 22-46. https://bit.ly/3vfa5fP

Rojas Valladares, A. L., Estévez Pichs, M. A., y Domínguez Urdanivia, Y. (2017). La práctica pre profesional: escenario propicio para promover los intereses profesionales en la Carrera de Educación Inicial, en la Universidad Metropolitana del Ecuador. Revista Conrado, 13(59), 43-50. https://bit.ly/2SmwhG

Ruilova Déleg, J. E. (2019). Las prácticas preprofesionales en la formación inicial docente de $E G B$, el rol del maestro tutor [Tesis de pregrado, Universidad de Cuenca]. https://bit.ly/3hLix2v

Sangrá Morer, A. (2001). Enseñar y aprender en la virtualidad. Educar, 1(28), 117-131. https://bit.ly/31CJPrL

Tamayo y Tamaño, M. (2012). El Proceso de la Investigación Científica. Limusa.

UNESCO. (2020). El Coronavirus COVID-19 y la educación superior: impacto y recomendaciones [Página web]. https://bit.ly/2QmQJG1

UNICEF. (2020). La falta de igualdad en el acceso a la educación a distancia en el contexto de la COVID-19 podría agravar la crisis mundial del aprendizaje [Página web]. https://uni.cf/3fJqEKe

Zambrano Acosta, J., Laurencio Leyva, A., y Millán Licea, M. R. (2018). La virtualidad como alternativa de formación universitaria. Revista didasc@lia: Didáctica y Educación, 9(2), 159-178. https://bit.ly/3fel2ZJ 